\title{
Low Grade Liver Dysplastic Nodule
}

National Cancer Institute

\section{Source}

National Cancer Institute. Low Grade Liver Dysplastic Nodule. NCI Thesaurus. Code C96770.

A hepatic dysplastic nodule characterized by the presence of low grade atypical cellular changes. 